\title{
Study on Bohai sea ice based on MODIS data
}

\author{
Zhou ye $^{1}$, Fan Zhaopeng ${ }^{1}$ \\ ${ }^{1}$ Naval air university, Yantai, China
}

\begin{abstract}
Based on MODIS L1B data, we identify information of sea ice area, peripheral line and density, and also use Landsat data with higher resolution to verify the results obtained from MODIS data. Using pseudo color processing technology is more intuitive and more clear to show the various information of the Bohai sea. The whole process of sea ice information extraction is described in detail from MODIS data downloads to image processing and analysis. The selected data basically cover the entire process from the initial ice age, the peak ice age to the final ice age. The ideal results are obtained, and it also shows that the MODIS satellite data is feasible to monitor sea ice, which provides a reference for the establishment of monitoring sea ice model.
\end{abstract}

\section{Introduction}

Sea ice directly affects changes in sea conditions, sea levels, atmospheric circulation and climate. As the only inland sea in China, the Bohai Sea is located in the middle latitude area, but the sea water freezes every winter. Monitoring the distribution and movement direction of sea ice is important to prevent and mitigate the sea ice disaster in winter.

Based on Terra and Aqua satellites, MODIS has features of high spatial resolution, wide vision and high radiation resolution. Terra and Aqua satellites complement each other in time and improve remote sensing data of sea ice in the Bohai sea. The present situation of China and the evolution of sea ice in Bohai sea have been preliminarily, scientifically and objectively evaluated and summarized.

\section{Theoretical basis and technical scheme}

\subsection{Spectral properties of sea ice in sea water}

Since the reflectance of sea ice and that of sea water are significantly different, the measurement results collected in the waveband from $0.4 \sim 2.4 \mu m$ indicate that the reflectance of sea ice changes throughout the season. The largest emissivity of snow-covered ice, annual thick ice and perennial ice is the range of $0.4 \sim 0.8 \mu \mathrm{m}$, reflectance decreases in the near infrared band, reaches the minimum at $1.6 \mu \mathrm{m}$, and then, at $1.9 \mu \mathrm{m}$ reaches a peak. The spectral reflectance of new ice is relatively low, ranging from 0.10 to $0.40 \mu \mathrm{m}$. The figure shows the average reflectance curves of ice, cloud and water in two different areas of the Bering sea in each band of the MAS. As the figure, the reflectance of ice and water in the range of $0.4 \sim 0.8 \mu \mathrm{m}$ is significantly different from that of ice and water.

\subsection{Technical solution}

Data from MODIS 1 and MODIS 2 bands are selected for processing. Both of them have a resolution of $250 \mathrm{~m}$ and are located in the visible and near infrared bands respectively. They have very good ability to distinguish in the aquatic and land.

By removing land to monitored the ocean, the visual effects is improved with image enhancement technology. The processed binary image is segmented into remote sensing images to eliminate the clutter, speckle and the interference caused by noise. The edge detection method is used to extract the outer edge of sea ice.The calculation of sea ice concentration is realized by using the single channel algorithm of inversion of sea ice concentration in Bohai sea.

\section{MODIS data preprocessing}

\subsection{Data download}

The MODIS data can be downloaded directly from NASA's official website, which facilitates this study. Here we take the remote sensing image of December 3, 2008 as an example. The original image is as follows: 


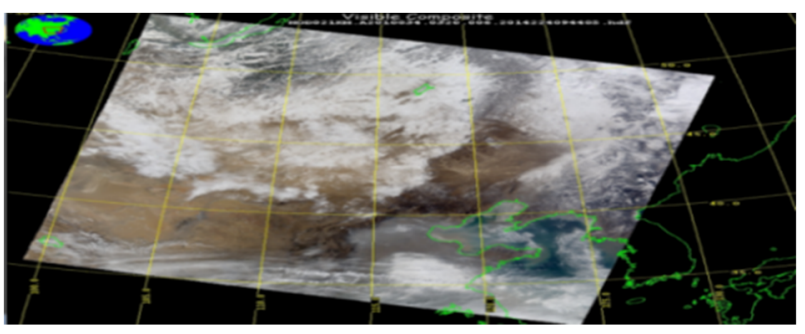

Fig.3-1.Original download

\subsection{Data preprocessing}

Data preprocessing part mainly uses ENVI software to complete. It is the ITT Visual Information Solutions and the company's flagship product, developed by scientists in the field of remote sensing using IDL a powerful remote sensing image processing software. Using ENVI to open the downloaded MODIS data, you can directly read the image information after calibration. After software geometric correction and correction of the bowknot effect, the result is as shown in the figure.

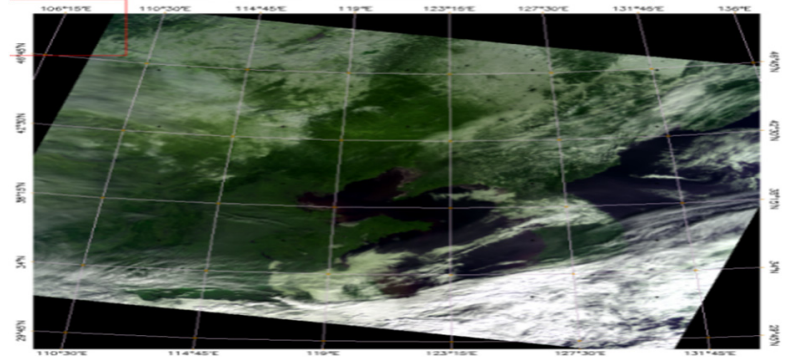

Fig.3-2. Data preprocessing

\section{MODIS image processing and analysis}

\subsection{Removal of terrestrial interference information (land mask)}

Because MODIS the 6th band land and sea water (including ice) radiation have a larger difference, make the decision tree, the radiation intensity is greater than or equal to 1 (land) set to 0 , the radiation degree is less than 1 (water, ice) set to 1 , and will the proceeds of binary figure and the required band "and" operation, mask, so that it will land information and keep the ocean information as shown in figure 4-1.

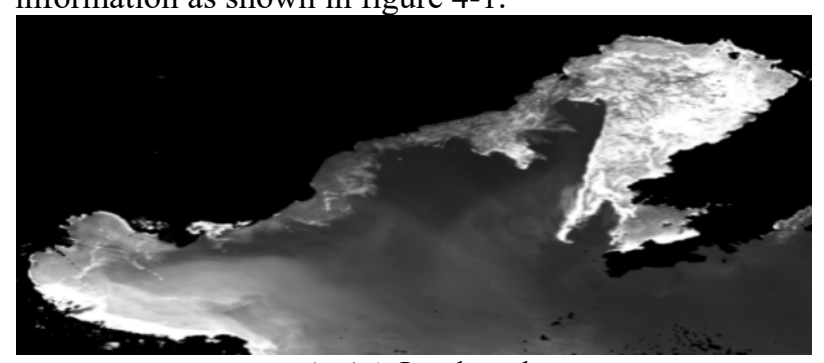

Fig.4-1. Land mask

\subsection{Ice water to identify}

In visible light range, the reflectivity of water is low in general, no more than $10 \%$, commonly at $4 \% \sim 5 \%$, and increases with the increasing of wavelength gradually reduce, at wavelength of $0.6 \mu m$ is about $2 \% \sim 3 \%$, when the wavelengths longer than $0.75 \mu \mathrm{m}$, the water becomes almost all of the absorber. In the remote sensing image of near infrared band, the clear water is dark black. Therefore, it is more accurate to extract water from the combination of near-infrared band and visible band, namely, the 2nd and 4th bands of MODIS.

By selecting the trough between the two peaks as the threshold value, the ice-water binary graph was obtained, and the decision tree was used to classify it to generate fig 4-6. Green, white and blue are the identified land, sea ice and sea water ${ }^{[3]}$.

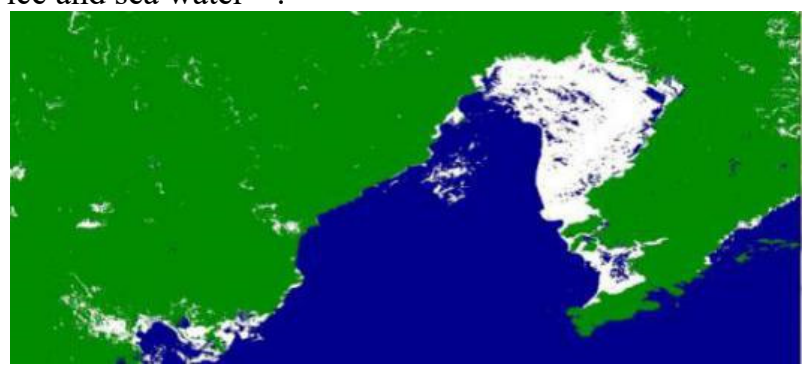

Fig.4-2. Ice water to identify

\subsection{Analysis of sea ice concentration}

The principle of article is sea ice concentration inversion algorithm of single channel, using the commonly used single channel system point method for sea ice intensity of inversion, the specific identification method is: collect like pixel's actual albedo, when albedo value of its lower than that of pure water, judged to be $0 \%$, when it is higher than pure ice albedo value in mixed pixels, judged to be $100 \%$, between which, according to the calculation of intensity. A is the actual albedo of the pixel, and represents the albedo values of pure ice and pure water in the mixed pixel respectively. The following equation is used to calculate the albedo of different channels:

$$
\rho_{\lambda}=\frac{\pi L_{\lambda} d^{2}}{E S U N_{\lambda} \cos \theta_{s}}
$$

where the subscript $\lambda$ represents different wavelength channels, $\rho_{\lambda}$ as the albedo, $L_{\lambda}$ is the spectral radiance at the point of the sensor entering the pupil after calibration, $d$ is the distance between the sun and the earth, $E S U N_{\lambda}$ is the average solar irradiance at the top of the atmosphere, $\theta_{s}$ is the solar zenith angle.

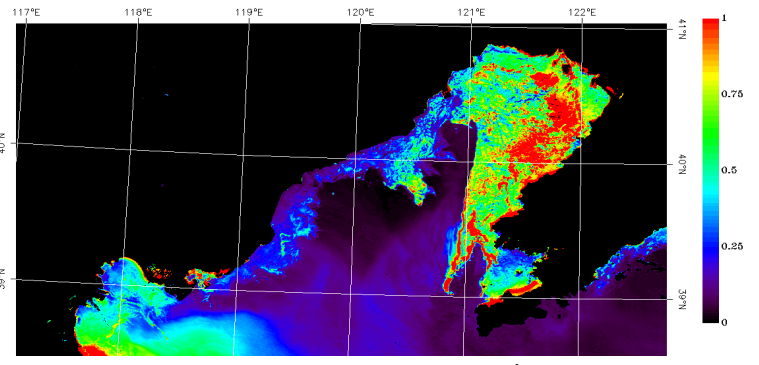

Fig.4-3. Ice concentratio 


\subsection{Sea ice margin}

Based on the existing ice water binary graph, the outer edge line of sea ice was obtained by feature extraction, image segmentation and edge detection algorithm.

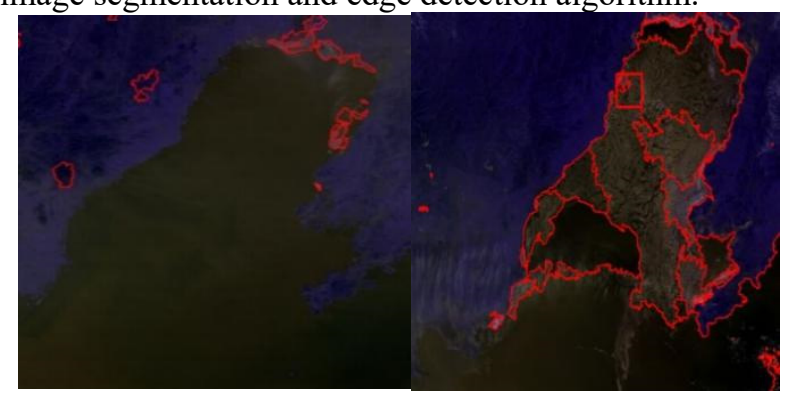

Fig.4-4. Freeze period and Glacial maximum

\section{Landsat data validation}

We use to identify the ice water ENVI processing Landsat data, with the same method as MODIS data. Through the establishment of interest areas, the threshold of ice and water is extracted and the binary image of ice and water is generated by using band operation. ENVI has the pixel statistics function, which can be used to calculate the pixel points of ice water. Through processing Landsat 8 a different band, get the ideal binary diagram of ice. The combined application of 4th, 5 th and 3th wavebands is more conducive to the separation between water body and continental boundary.

\subsection{Sea ice concentration test}

We select period of the Landsat images with MODIS data at the same time, in the area of the same size (the image cropping) is used to calculate sea ice area difference in two kinds of image data, to verify the accuracy of the MODIS data processing.Due to the obvious distinction between land and water in the 5th band, the interference factors of land are excluded by the 5th band. The decision tree was used to classify colors and counts the number of pixel points of ice.

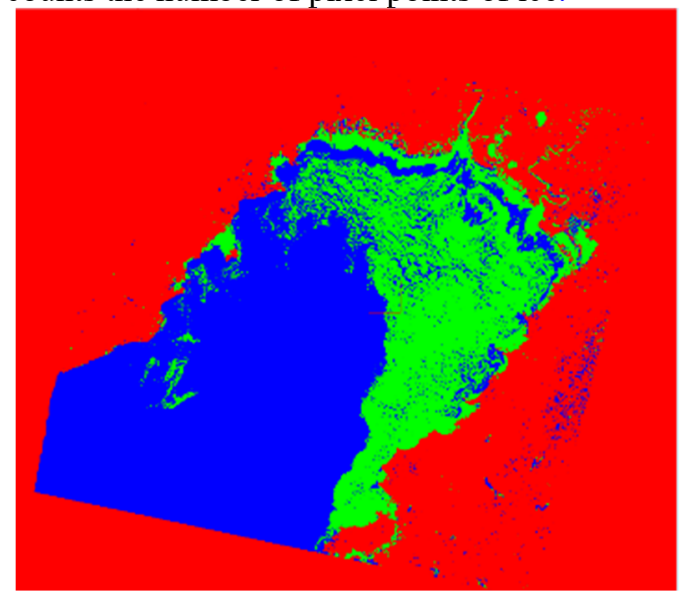

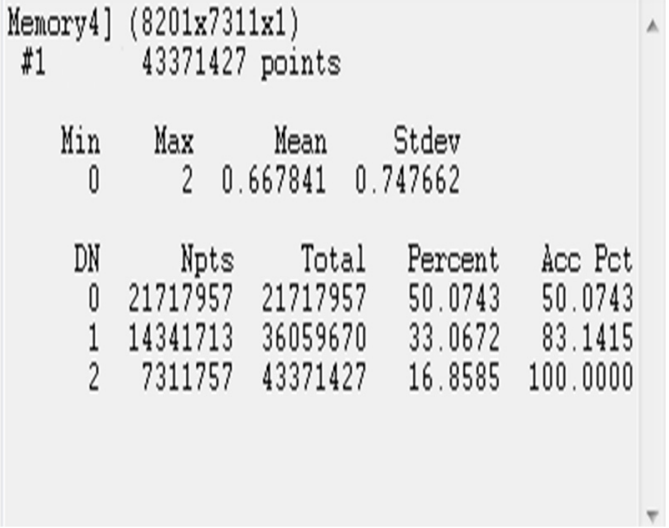

Fig.5-1.Pixel count

The ice area is about $16.86 \%$. Combined with total pixel points, the ice water area can be calculated separately. Using this method, the intensity information of 20 points in MODIS data is randomly verified by random pixel point comparison. 20 points are randomly selected from the intensity image of MODIS and the information is recorded. The corresponding range of latitude and longitude information is searched on the langdsat image, and the percentage of sea ice area in the statistical range is verified by the comparison between the two. Through verification, the statistical results of all points are as follows:

Table 1. Pixel contrast.

\begin{tabular}{|l|c|c|c|c|c|c|c|c|c|c|}
\hline Number & 1 & 2 & 3 & 4 & 5 & 6 & 7 & 8 & 9 & 10 \\
\hline MODIS & 0.54 & 0.80 & 0.73 & 0.92 & 0.76 & 0.64 & 0.51 & 0.61 & 0.21 & 0.76 \\
\hline Landsat & 0.55 & 0.81 & 0.71 & 0.91 & 0.74 & 0.67 & 0.54 & 0.65 & 0.37 & 0.75 \\
\hline Number & 11 & 12 & 13 & 14 & 15 & 16 & 17 & 18 & 19 & 20 \\
\hline MODIS & 0.84 & 0.91 & 0.76 & 0.35 & 0.62 & 0.74 & 0.37 & 0.02 & 0.75 & 0.64 \\
\hline Landsat & 0.86 & 0.94 & 0.74 & 0.39 & 0.61 & 0.75 & 0.41 & 0.00 & 0.77 & 0.60 \\
\hline
\end{tabular}

Through analysis, the error of sea ice concentration obtained from MODIS data and Landsat data with higher resolution is $2.73 \%$, which meets the application requirements.

\section{Conclusion}

This article briefly introduces MODIS L1B of data preprocessing.In this paper, all images are calibrated to uniform precision in the same projection mode and projection coordinate system.It lays a foundation for using unified land template.According to the characteristics of MODIS data channels, appropriate channels are selected for cloud detection to remove the cloud area. Then, according to the difference of ice, water brightness and reflectance, the recognition threshold of ice water is obtained and the ice water is discriminated. The single channel point method was used for the inversion of sea ice concentration, and Landsat data are used to verify the treatment results. The conclusions in this paper are basically consistent with the actual situation and provide reliable data for better monitoring of sea ice, preventing and controlling disasters in the future. 


\section{Reference}

1. Han Suqin. EOS/MODIS satellite observations of sea ice in the Bohai sea $[\mathrm{J}]$. Meteorological science. 2005(06).

2. Wu Kuiqiao. Application of MODIS data in sea ice remote sensing [J]. Marine forecast. 2005(S1).

3. Guo Qiaozhen, $\mathrm{Gu}$ Wei. A revised model of sea ice area extraction in Bohai sea based on remote sensing data [J]. Marine science. 2008(08).

4. Guo Qiaozhen. System's Establishment and Applied Research of Bohai Sea Ice Area Information [J], Sea Notification, 2007(26).

5. Gu Wei,Xie Feng. The spatial distribution characteristics of winter sea ice resources in liaodong bay [J], Resources science,2003(03). 